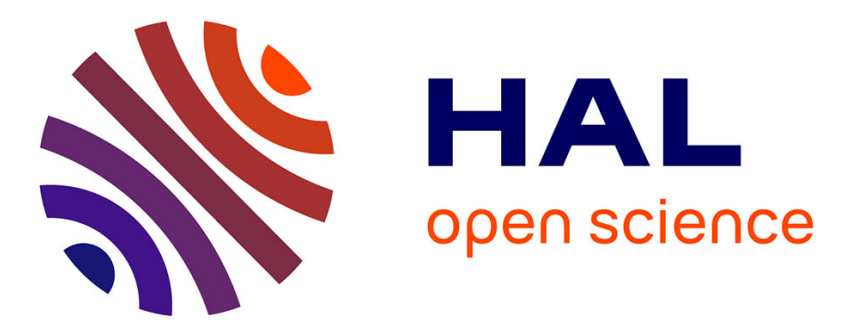

\title{
Laser Spectroscopy with Nanometric Cells Containing Atomic Vapor of Metal: Influence of Buffer Gas.
}

D. Sarkisyan, G. Hakhumyan, A. Sargsyan, R. Mirzoyan, Claude Leroy, Y. Pashayan-Leroy

\section{> To cite this version:}

D. Sarkisyan, G. Hakhumyan, A. Sargsyan, R. Mirzoyan, Claude Leroy, et al.. Laser Spectroscopy with Nanometric Cells Containing Atomic Vapor of Metal: Influence of Buffer Gas.. 16th International School on Quantum Electronics: Laser Physics and Applications, Sep 2010, Nessebar, Bulgaria. pp.77470C, 10.1117/12.881838 . hal-00620853

\section{HAL Id: hal-00620853 https://hal.science/hal-00620853}

Submitted on 8 Sep 2011

HAL is a multi-disciplinary open access archive for the deposit and dissemination of scientific research documents, whether they are published or not. The documents may come from teaching and research institutions in France or abroad, or from public or private research centers.
L'archive ouverte pluridisciplinaire HAL, est destinée au dépôt et à la diffusion de documents scientifiques de niveau recherche, publiés ou non, émanant des établissements d'enseignement et de recherche français ou étrangers, des laboratoires publics ou privés. 


\title{
Laser Spectroscopy with Nanometric Cells Containing Atomic Vapor of Metal: Influence of Buffer Gas
}

\author{
D. Sarkisyan ${ }^{\mathrm{a}}$, G. Hakhumyan ${ }^{\mathrm{a}, \mathrm{b}}$, A. Sargsyan ${ }^{\mathrm{a}}$, R.Mirzoyan ${ }^{\mathrm{a}}$, C. Leroy ${ }^{\mathrm{b}}$, Y. Pashayan-Leroy ${ }^{\mathrm{b}}$ \\ ${ }^{a}$ Institute for Physical Research, NAS of Armenia, Ashtarak, 0203, Armenia \\ ${ }^{b}$ Laboratoire Interdisciplinaire Carnot de Bourgogne, UMR CNRS 5209 - Université de Bourgogne, \\ F-21078 Dijon Cedex, France
}

\begin{abstract}
Comparison of absorption and fluorescence in a nano-cell containing $\mathrm{Rb}$ vapor with other $\mathrm{Rb}$ nano-cells where neon gas is added is presented. The effect of collapse and revival of Dicke-type narrowing is still observable for the Rb nano-cells where Ne gas is added under 6 and 20 Torr pressure for the thickness $L=\lambda / 2$ and $L=\lambda$, where $\lambda$ is a resonant laser wavelength $794 \mathrm{~nm}\left(\mathrm{D}_{1}\right.$ line). Particularly for 6 Torr the line-width of the transmission spectrum for the thickness $L=\lambda / 2$ is 2 times narrower than that for $L=\lambda$. For an ordinary $\mathrm{Rb}$ cell with $L=0.1-10 \mathrm{~cm}$ with addition of buffer gas, the velocity selective optical pumping/saturation (VSOP) resonances in saturated absorption spectra are fully suppressed when the buffer gas pressure $>0.5$ Torr. A spectacular difference is that for $L=\lambda$, VSOP resonances located at the atomic transition are still observable even when Ne pressure is $\geq 6$ Torr. Narrowband fluorescence spectra of the nano-cell with $L=\lambda / 2$ can be used as a convenient tool for online buffer gas pressure monitoring for the conditions when ordinary pressure gauges are unusable. Comparison of electromagnetically induced transparency (EIT) effect in a nano-cell filled with pure $\mathrm{Rb}$ with another nano-cell, where buffer gas nitrogen is added, is presented. Use of gas $\mathrm{N}_{2}$ inside the $\mathrm{Rb}$ nanocell strongly extended the region of the coupling laser detuning where EIT resonance is still possible to form.
\end{abstract} transparency

Keywords: Atomic spectroscopy; nano-cell; buffer gas; coherent narrowing, electromagnetically induced

\section{INTRODUCTION}

A number of works published in recent years deal with the laser spectroscopy of the resonance absorption and fluorescence of an atomic vapor column of 10-100 $\mu \mathrm{m}$ thickness [1,2] and of an atomic vapor column enclosed in socalled extremely thin cells (ETC), or nano-cells $[3,4]$ filled with pure metallic Cesium and/or Rubidium. It was shown in the works [5-13] that an important parameter determining the spectral width, the shape of the line, and the absorption in these nano-cell, is the ratio $L / \lambda$ with $L$ being the thickness of the vapor column (i.e. the distance between the nano-cell windows) and $\lambda$ is the wavelength of the laser radiation resonant with the corresponding atomic transition of $\mathrm{Cs}$ or $\mathrm{Rb}$, $\mathrm{D}_{2}$ and $\mathrm{D}_{1}$ lines. In particular it was shown that the spectral width of the resonant absorption reaches its minimum value at $L=(2 \mathrm{n}+1) \lambda / 2$ ( $\mathrm{n}$ is an integer); this effect has been termed the Dicke coherent narrowing (DCN) effect. Note that the spectral width of the resonant absorption reaches its absolute minimum value for $\mathrm{L}=\lambda / 2$ which is more than 4 times narrower than the Doppler width, recorded in the cells of the conventional length (CCL) $0.1-10 \mathrm{~cm}$ (note that the Doppler width of hyperfine transition in CCL is about of $350 \mathrm{MHz}$ for $\mathrm{Cs}$ and $\sim 500 \mathrm{MHz}$ for the $\mathrm{Rb}$, at room temperature). Relative contrast of spectral width decreases with respect to the broad pedestal which increases proportionally to $\mathrm{L}=3 \lambda / 2,5 \lambda / 2,7 \lambda / 2$ etc. Simple calculation shows that the the Dicke coherent narrowing should be observable up to the thickness $\mathrm{L}_{\max }=\mathrm{V} t$, where $\mathrm{V}$ is the mean thermal velocity of Cs $/ \mathrm{Rb}$ atoms $(\sim 250 / 200 \mathrm{~m} / \mathrm{s})$, and the $t$ is excited state lifetime $(\sim 30 / 27 \mathrm{~ns})$, that is $\mathrm{L}_{\max } \sim 7.5 / 5.4 \mu \mathrm{m}$ for the $\mathrm{Cs} / \mathrm{Rb}$ atomic vapor, correspondingly.

It was also demonstrated [8-13] that for $\mathrm{L}=\mathrm{n} \lambda$ the spectral width of the resonant absorption reaches its maximum value, close to the Doppler width, recorded in the CCL. This phenomenon was termed collapse of DCN. For relatively high pump intensity $\left(>1 \mathrm{~mW} / \mathrm{cm}^{2}\right)$, the VSOP resonances of a reduced absorption (with the line-width close to the natural one), and centered on the hyperfine transitions occurs sharply when $L=\mathrm{n} \lambda$, while the narrowest and well-marked VSOP resonance occurs when $L=\lambda$. There are several distinct benefits to use these VSOP resonances as a frequency reference for atomic transitions of alkali atoms in comparison with widely used Saturation Absorption (SA) technique. Particularly, use of the nano-cell with the thickness $\mathrm{L}=\lambda$ allows the complete elimination of crossover $(\mathrm{CO})$ resonances when $\mathrm{L}=\lambda$. " $\lambda$-Zeeman technique" (LZT) is shown to be efficient for studies of individual transitions between the Zeeman sublevels of hyperfine levels in an external magnetic field of $1-5000 \mathrm{G}$ [14]. LZT is based on nano-cell with 
thickness $L=\lambda$. Narrow VSOP resonances in the transmission spectrum of the ETC are split into several components in a magnetic field; their frequency positions and probabilities depend on the B-field .

In contrast to the transmission spectra, when $L$ increases, the spectral width of the fluorescence spectra increases monotonically, without oscillations of the line-width versus $L$, while remaining below the Doppler broadening even when the thickness of the vapor column increased up to a 3-4 $\mu \mathrm{m}[3,4,7-9,13]$. The narrowest line-width of the fluorescence spectrum is obtained when $L$ is in the range $\lambda / 4<L \leq \lambda / 2$ and is 1.5-1.8 times narrower than the absorption spectral width for $\mathrm{L}$ in the same range. Another difference between absorption and fluorescence processes obtained with the nano-cells is that VSOP resonances of a reduced fluorescence appear at much higher intensity $\left(>20 \mathrm{~mW} / \mathrm{cm}^{2}\right)$. The " $\lambda / 2$-Zeeman technique" (HLZT) is shown to be efficient for studies of individual transitions between the Zeeman sublevels of hyperfine levels in an external magnetic field of $10-2500$ G. HLZT is based on resonant fluorescence spectrum emitted by a nano-cell with $L=\lambda / 2[15,16]$.

Considerable interest of coherent population trapping (CPT) and the related electromagnetically induced transparency phenomena is caused by a number of important applications in a variety of fields such as laser cooling, information storage, magnetometry, spectroscopy, atomic frequency references etc [17 - 19]. EIT resonance can occurs in $\Lambda$-system with a two long-lived states and one excited state coupled by two laser fields (so called coupling and probe lasers). From the application point of view it is important to reduce dimensions of the cell which are containing atomic vapor of metal where an EIT resonance is formed, while keeping resonance parameters good (such as narrow line-width and contrast)[20-24].

We have developed a new nano-cells filled with the natural $\mathrm{Rb}$ where neon gas is added under 6 Torr and 20 Torr pressure [25]. Also, the nano-cell filled with the natural $\mathrm{Rb}$ where nitrogen gas is added is fabricated. Comparison of the above mentioned processes in nano-cell filled with pure $\mathrm{Rb}$ is provided both experimentally and theoretically.
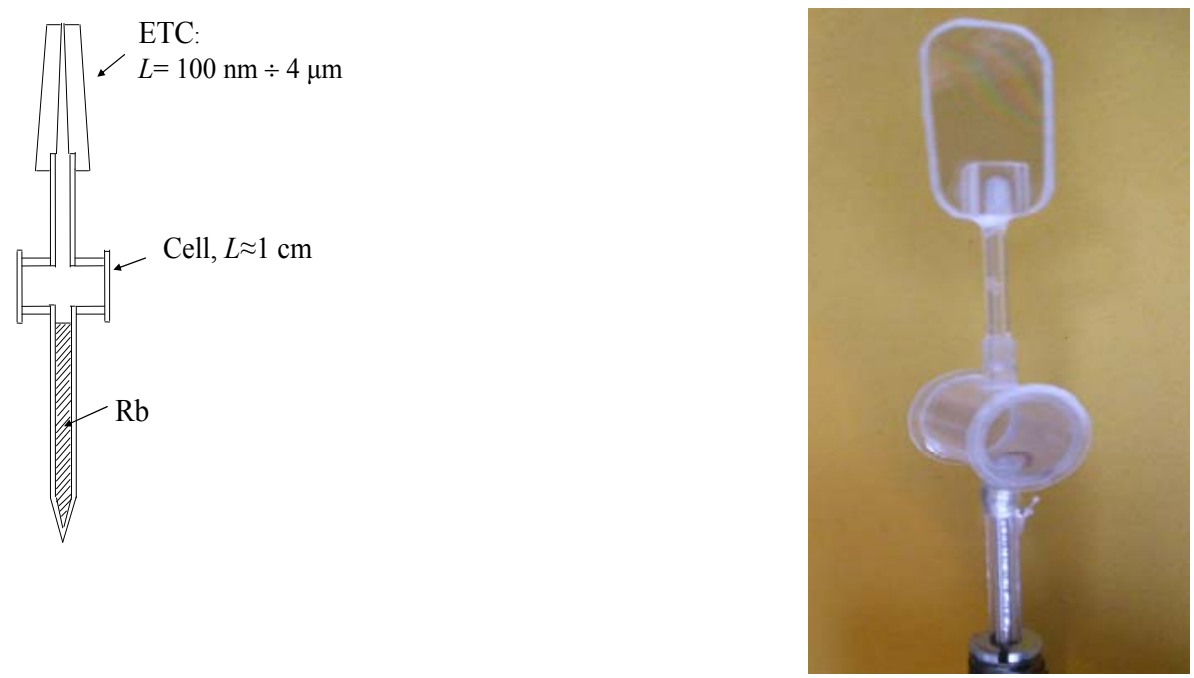

Fig.1 MRC consisting of 2 interconnected regions: ETC or nano-cell (shown in the upper part) with wedged thickness in the range of $100 \mathrm{~nm}-4 \mu \mathrm{m}$, and $1 \mathrm{~cm}$-long sapphire cell (shown the lower part) with the sapphire windows (the sapphire plates-windows are cut across the $\mathrm{c}$-axis). MRC is filled with the natural $\mathrm{Rb}$ with addition of neon gas at 6 Torr or 20 Torr pressure.
Fig.2 Photography of the multi-region cell. The nano-cell is seen in the upper part, while $\sim 1 \mathrm{~cm}-$ long sapphire cell is shown in the middle part. MRC is fixed inside the oven with the help of the aluminum tube-holder which is tied to the side arm (only the top is seen at the bottom of the photo).

\section{EXPERIMENTAL}

\section{II.1. The design of multi- region cells filled with the $\mathrm{Rb}$ and neon gas}


Multi-region cell consists of two interconnected cells and a side-arm containing metal. The nano-cell region (shown in the upper part of Fig.1) has a wedged thickness of the gap between the windows that allows one to exploit atomic vapor column thickness in the range of $0.1-4 \mu \mathrm{m}$. The first design of the nano-cell (or so called extremely thin cell), which consists of windows and a vertical side-arm (a metal reservoir), was presented in [3]. Later, this design has been somewhat modified; the typical example of the last version is presented in Fig.1. We use $15 \times 25 \mathrm{~mm}$ rectangular window wafers, fabricated either from commercial sapphire or garnet $\left(\mathrm{Y}_{3} \mathrm{Al}_{5} \mathrm{O}_{12}\right)$ crystal, which are both chemically resistant to the chemically aggressive vapors of alkali metals. In order to minimize the birefringence effect, the sapphire plates are cut across the c-axis, while the garnet is free of birefringence. The wafers have to be perfectly polished (better than $\lambda / 20$ ) before the use. In order to form the wedge gap in the vertical direction (this is needed to have a variable thickness of atomic vapor column-see the inset in Fig.1), an $\mathrm{Al}_{2} \mathrm{O}_{3}$ strip, $\sim 1000 \mathrm{~nm}$ thick, $5 \mathrm{~mm}$ long, and $1 \mathrm{~mm}$ wide, is deposited on the surface of one of the windows in its lower part (in some cases, a platinum strip is used as a spacer: Pt is also resistant to highly corrosive hot $\mathrm{Rb}$ atomic vapor). Prior to the deposition, a $2 \mathrm{~mm}$ hole is drilled in the bottom of the windows, into which a $25 \mathrm{~mm}$ long tube of the same diameter, made of commercial sapphire, is inserted. With the help of this sapphire tube (inside diameter is of $1 \mathrm{~mm}$ ) the nano-cell is connected with the $1 \mathrm{~cm}$-long cell region (the lower part of Fig.1) with 2 sapphire windows. The $1 \mathrm{~cm}$-long sapphire cell with sapphire windows is terminated by with a 4-cm long sapphire side-arm. Then, the entire construction is assembled and glued in a vacuum furnace. After the gluing, a glass extension is sealed to the sapphire tube, and the multi-region cell is filled with a natural mixture of the ${ }^{85} \mathrm{Rb}(72 \%)$ and ${ }^{87} \mathrm{Rb}(28 \%)$ as well as with a buffer gas neon, as is done for an ordinary glass cell. Photograph of the multi-region cell is presented in Fig.2. The holder of the multi-region cell (which is tied to the side arm and is seen at the bottom in Fig.2) is made from the short aluminium tube. With the help of this holder MRC is fixed inside the oven.

We have used 2 MRCs, filled with 6 Torr and 20 Torr of neon gas. Such design of MRC allows one to compare absorption and fluorescence signals simultaneously recorded by the nano-cell and $1 \mathrm{~cm}$-long ordinary cell.

The MRC is placed in the oven with openings, which allow passing the laser radiation and registration of fluorescence in the direction perpendicular to the laser beam. The temperature of the MRC was kept at $\sim 120{ }^{\circ} \mathrm{C}$ at the side-arm (the latter defines $\mathrm{Rb}$ atomic vapor pressure), and somewhat $\left(20^{\circ} \mathrm{C}\right)$ higher at the windows in order to prevent $\mathrm{Rb}$ vapor condensation at the windows. This regime corresponds to the number density of $\mathrm{Rb}$ atoms $N \sim 10^{13}$ at $/ \mathrm{cm}^{3}$. To measure the transmission and fluorescence spectra at different nano-cell thicknesses, the oven with MRC was smoothly translating vertically as indicated by an arrow in Fig.3.

\section{II.2. Experimental arrangement for spectroscopic measurements}

The experimental arrangement for transmission and fluorescence measurements is presented in Fig.3. Extended cavity diode laser (ECDL) with $\lambda=794 \mathrm{~nm}, \sim 30 \mathrm{~mW}$ power and spectral width $\sim 1 \mathrm{MHz}$ was used. Faraday isolator (FI) was used in order to avoid optical feedback. Diode laser radiation is focused on the cell (2) nearly perpendicular to its window

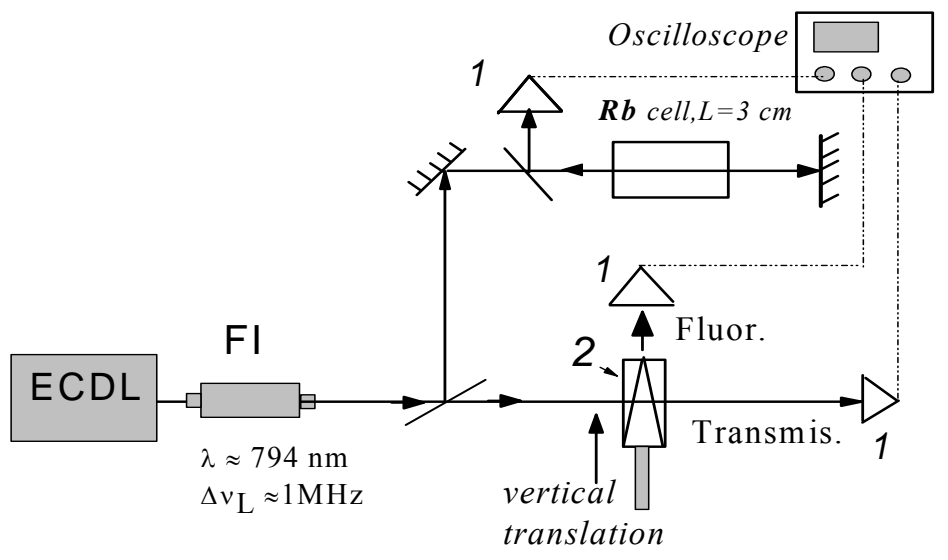

Fig.3. Sketch of the experimental setup. $E C D L$ diode laser with $\lambda=$ $794 \mathrm{~nm}$; FI- Faraday isolator, 2multi-region cell (inside the oven), or an auxiliary nano-cell filled with pure $\mathrm{Rb}$ or $\mathrm{Cs}$; the upper part - unit for the frequency reference formation, 1 photodiodes.

surface (this is very important in the case of nano-cell use[3,7]), with a spot size of $\varnothing \sim 0.5 \mathrm{~mm}$. The MRC is placed in the oven made from non-magnetic materials, which has 3 openings: 2 for the laser beam transmission and one for 
registration of resonance fluorescence in perpendicular direction to the laser beam. The temperature of the ETC is kept at $\sim 110-120^{\circ} \mathrm{C}$ on the side arm (this defines the $\mathrm{Rb}$ atomic vapor pressure), and $20-30{ }^{\circ} \mathrm{C}$ higher at the windows (in order to prevent the $\mathrm{Rb}$ vapor condensation at the windows). This regime corresponds to the number density of Rb atoms $N \sim$ $10^{13} \mathrm{at} / \mathrm{cm}^{3}$. In order to measure the transmission and fluorescence spectra at different nano-cell thicknesses, the oven with the MRC was smoothly moved vertically as indicated by an arrow in Fig.3.

In order to obtain the transmission or/and fluorescence spectra, the laser radiation frequency is linearly scanned in an appropriate spectral region around the studied transition group of either the ${ }^{85} \mathrm{Rb}$ or ${ }^{87} \mathrm{Rb}, \mathrm{D}_{1}$ line. Part of radiation $(\sim 50 \%)$ is branched to auxiliary reference setup (the upper part in Fig.3) for comparison with the spectra obtained in the main nano-cell. Depending on task, it could be: i) resonant transmission or fluorescence spectra obtained with $3 \mathrm{~cm}-$ long $\mathrm{Rb}$ cell; ii) SA spectra based on a room temperature $3 \mathrm{~cm}$ - long Rb cell; iii) transmission spectra of hyperfine atomic transitions in an auxiliary nano-cell, which is preferable in some cases. The spectra were recorded by photo-detectors (1) followed by a digital four channel storage oscilloscope, Tektronix TDS 2014B.

\section{II.3. Key features of resonant absorption in nano-cell filled with pure alkali metal (Cs)}

We note that in $[3,7,910,13]$ the resonance absorption at $\mathrm{D}_{2}$ lines of $\mathrm{Rb}$ and $\mathrm{Cs}$ atoms has been studied where the frequency distance between the upper hyperfine sublevels is rather small (several tens of $\mathrm{MHz}$ ), which hinders practically the consideration of behavior of separate atomic transitions. From this point of view, the $\mathrm{D}_{1}$ lines of Cs is more convenient because the frequency separation of the upper hyperfine levels reaches $\sim 1.167 \mathrm{GHz}$ (see Fig.4) and hence the atomic transitions are completely resolved. In order to demonstrate the key features of resonant absorption in nano-cell, an auxiliary nano-cell filled with pure Cs was used.

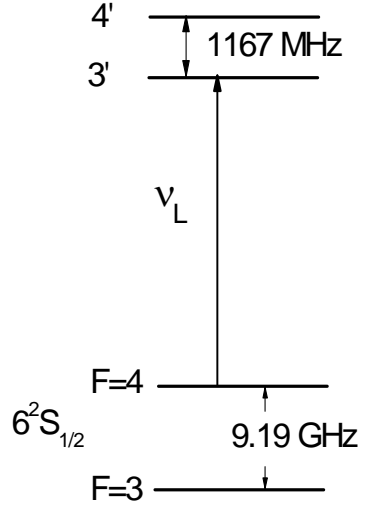

Fig.4 Relevant energy levels of the Cs, $\mathrm{D}_{1}$ line.

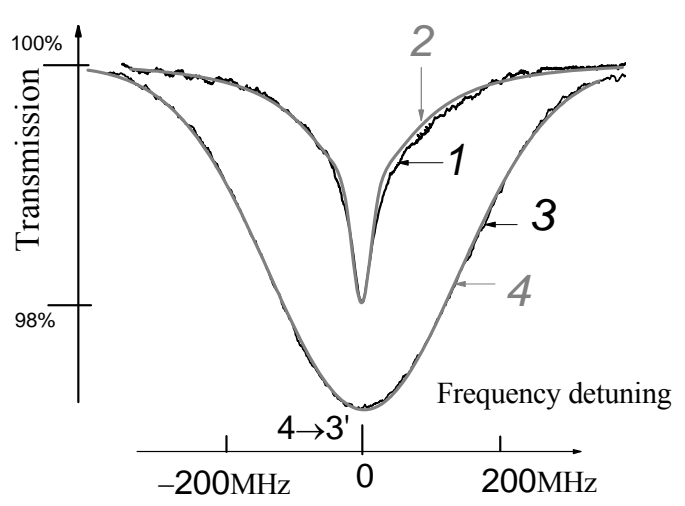

Fig.5. Transmission spectra, $4 \rightarrow 3$ ' , the nano-cell with $\mathrm{L}=\lambda / 2$ and the cell with $\mathrm{L}=3 \mathrm{~cm}$; (1) transmission spectrum of the nano-cell which is well described by curve (2) the "pseudoVoigt2" function with FWHM 75 MHz; (3) Doppler-broadened transmission spectrum from the $3 \mathrm{~cm}$-long cell which is well described by (4) the Gaussian profile with FWHM $350 \mathrm{MHz}$.

The laser frequency was scanned in the range of $\sim 10 \mathrm{GHz}$ covering all four spectral components $F_{\mathrm{g}}=3 \rightarrow F_{\mathrm{e}}=3 ; 4$ and $F_{\mathrm{g}}=4 \rightarrow F_{\mathrm{e}}=3 ; 4$ of the hfs of the $\mathrm{Cs} \mathrm{D}_{1}$ line (subscripts "g" and "e" denote, respectively, the lower and upper levels of the transition). In order to obtain the transmission spectra for different thicknesses, the oven with the nano-cell was moved vertically as indicated by an arrow in Fig. 3. A small portion of radiation was directed onto a cell of $3 \mathrm{~cm}-$ long at room temperature where the well-known saturated absorption (SA) scheme was realized. The filters F were also used for attenuation of the probe (as well as pump) beam in the SA scheme in order to obtain a reference spectrum with a width close to natural.

Depicted in Fig. 5 are the transmission spectra of the nano-cell and of the cell with $L=3 \mathrm{~cm}$ for atomic transition $F_{\mathrm{g}}$ $=4 \rightarrow F_{\mathrm{e}}=3$ and the thickness of the nano-cell $L=\lambda / 2$. Laser intensity $\sim 30 \mu \mathrm{W} / \mathrm{cm}^{2}$. Curve (1) is the transmission spectrum of the nano-cell at side-arm temperature $\sim 110^{\circ} \mathrm{C}$. The absolute value of absorption amounts to $\sim 2 \%$. The experimental profile 1 of the transmission line for $L=\lambda / 2$ is best approximated by the curve 2 which is described by the 
"pseudo-Voigt2" function of the Origin-7 program with the Lorentzian and the Gaussian profiles having the FWHM 70 and $77 \mathrm{MHz}$, respectively (the parameters $m$ and $A$ of the "pseudo-Voigt2" function have also been adjusted). Curve 2 has FWHM $\sim 75 \mathrm{MHz}$. Curve 3 is the experimental Doppler-broadened transmission spectrum of the cell with $L=3 \mathrm{~cm}$ which is well described by the Gaussian profile 4 with FWHM $\sim 350 \mathrm{MHz}$ (curves 3 and 4 in Fig. 5 coincide practically). So, for $L=\lambda / 2$ a 4.6 times narrowing of the spectrum is observed. But if we compare the transmission spectra from the nanocell and the usual cell at the temperature of windows of both of them equal $\sim 150^{\circ} \mathrm{C}$, the $\mathrm{FWHM}$ is $430 \mathrm{MHz}$ and the spectrum narrowing for $L=\lambda / 2$ is 5.7 times.

Figure 6 shows the transmission spectra for the same atomic transition and the thickness varying from $\lambda / 2$ to $7 \lambda / 2$ with the step of $\lambda / 2$ at the laser intensity $\sim 0.1 \mathrm{~mW} / \mathrm{cm}^{2}$. The phenomena of DCN and collapse of DCN are well seen in Fig. 6: the minimum value of the spectral width is reached at the thicknesses $L=(2 n+1) \lambda / 2$. Note that for $L=\lambda$ / 2 the width is minimal: $75-80 \mathrm{MHz}[5,6,10]$, whereas for $L=3 \lambda / 2,5 \lambda / 2$, and $7 \lambda / 2$, the narrowing of the spectrum occurs in the presence of increasing broad Doppler pedestal). As seen in Fig. 6, for $L=n \lambda$ the spectral width is maximal (for $L=\lambda$ it is $\sim 300 \mathrm{MHz}$ ). So, fourfold narrowing of the transmission spectrum takes place for $L=\lambda / 2$ as compared to $L=\lambda$. Note that in [6] the twofold narrowing of the spectrum has been observed which may probably be explained as follows: because of small gaps between the nano-cell windows the evacuation of residual gases (adsorbed at the window surfaces) is strongly hindered and hence many-hours evacuation is needed at the temperature of $\sim 400^{\circ} \mathrm{C}$; work [6] used the first nano-cell model where the evacuation of residual gases was performed worse than in subsequent models. Residual gases may lead to an additional broadening of the transmission spectrum and this influence is more for the thickness $L=\lambda / 2$ than for $L=\lambda$. Note that the peak transmission for the thickness $L=\lambda / 2$ is practically equal to the peak transmission for $L=\lambda$, whereas at the increase in laser intensity the peak transmission for $L=\lambda / 2$ becomes higher than for $L=\lambda$. The lower curve in Figs.6, 7 are obtained with the standard SA technique from the cell of usual length.

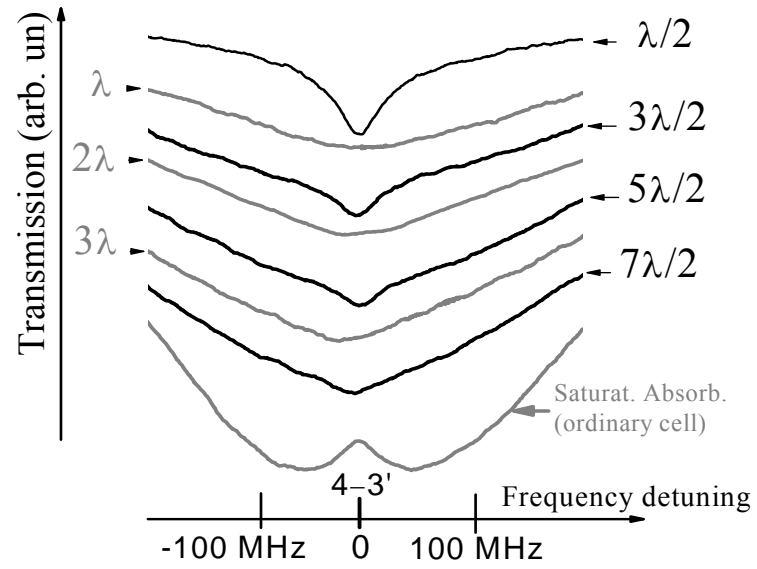

Fig. 6. Transmission spectra, 4' $\rightarrow$ 3' for variation of the column thickness from $\lambda / 2$ to $7 \lambda / 2$ with the step of $\lambda / 2$; laser intensity $\sim 0.1 \mathrm{~mW} / \mathrm{cm}^{2}$. The phenomena of DCN and collapse of DCN are well seen; the lower curve is obtained with the standard SA technique from the cell with $L=3 \mathrm{~cm}$. Spectra are shifted vertically for the convenience.

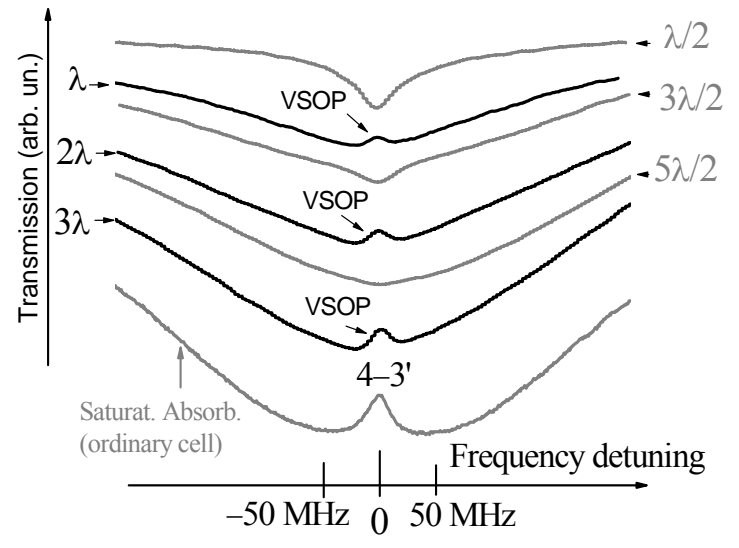

Fig.7. Transmission spectra. All parameters are the same as in Fig. 6, but the laser intensity is $\sim 5$ $\mathrm{mW} / \mathrm{cm}^{2}$ Theretical model presented in $[5,8,12]$ well described experimental curves shown in Fig.6 and Fig.7. Spectra are shifted vertically for the convenience.

\section{Resonant absorption and fluorescence in nano-cell filled with $\mathbf{R b}$ and neon gas.}

\section{III.1Resonant absorption in nano-cell filled with $\mathrm{Rb}$ and neon gas.}

Transmission spectra for ${ }^{85} \mathrm{Rb} \mathrm{D}_{1}$ line $3 \rightarrow 2^{\prime}, 3^{\prime}$ and ${ }^{87} \mathrm{Rb}, 2 \rightarrow 2^{\prime}$ transitions recorded in nano-cell filled with pure $\mathrm{Rb}$ (the cases $L=\lambda / 2$ and $L=\lambda$ ) are shown in Fig. 8 a for laser intensity $<0.1 \mathrm{~mW} / \mathrm{cm}^{2}$. Dicke-type coherent narrowing effect is well seen: the spectral line-width for $L=\lambda / 2(\sim 150 \mathrm{MHz})$ is narrower by factor of 3 than that for $L=\lambda(\sim 450 \mathrm{MHz})$. Transmission spectra for the nano-cell filled with $\mathrm{Rb}$ and 6 Torr of Ne are shown in Fig.8b for the same thicknesses. 


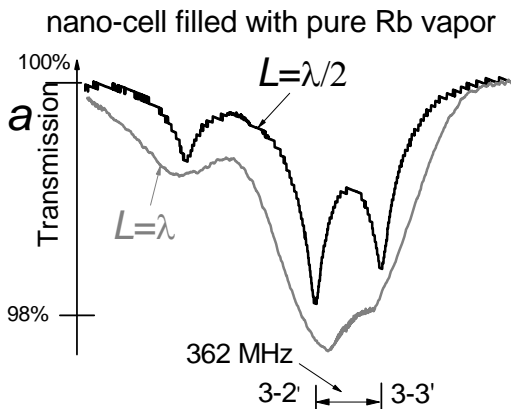

Laser frequency detuning

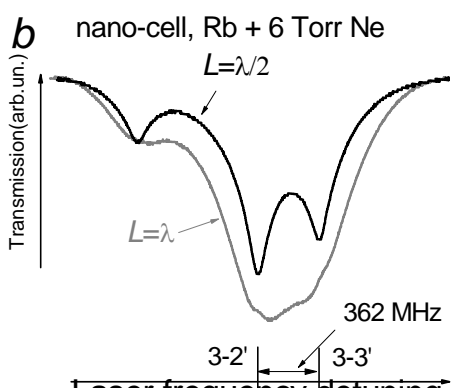

Laser frequency detuning

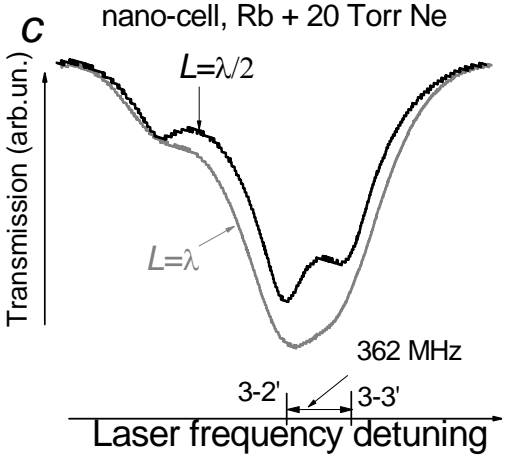

Laser frequency detuning

Fig.8. Transmission spectra for $L=\lambda / 2$ and $L=\lambda$. a) nano-cell with pure ${ }^{85} \mathrm{Rb}$, DCN and its collapse is well seen; b) nano-cell with $\mathrm{Rb}+6$ Torr Ne, DCN and its collapse is still well seen; c) nano-cell with $\mathrm{Rb}+20$ Torr Ne, DCN and its collapse is yet seen, though less pronounced compared with a), b).

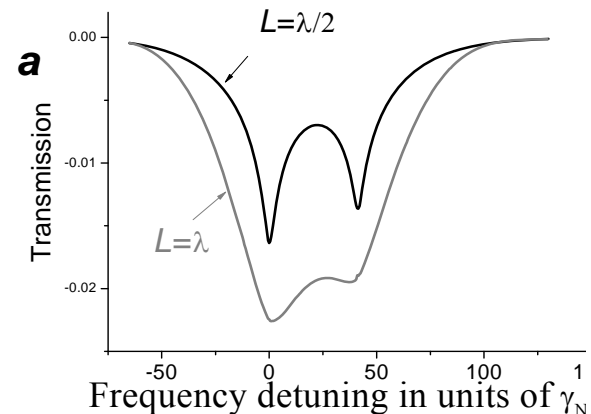

Frequency detuning in units of ${ }^{-50}{ }_{\mathrm{N}}^{1}$

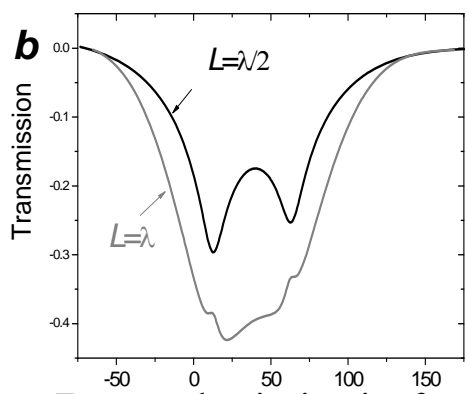

Frequency detuning in units of $\gamma_{\mathrm{N}}$

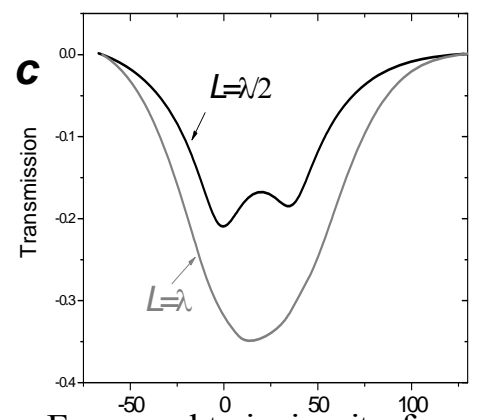

Frequency detuning in units of $\gamma_{N}$

Fig.9 Calculated spectra for cases presented in Fig.8. ${ }^{85} \mathrm{Rb}, \mathrm{D}_{1}$ line, only $3 \rightarrow 2$ ', 3 ' transitions a) pure $\mathrm{Rb}, L=\lambda / 2$, and $\left.\lambda, \Omega_{\mathrm{L}} \sim 0.04 \gamma ; \mathrm{b}\right) \mathrm{Rb}+6$ Torr Ne for $L=\lambda / 2$ and $\left.\lambda \Omega_{\mathrm{L}} \sim 0.4 \gamma ; \mathrm{c}\right) \mathrm{Rb}+20$ Torr Ne, for $L=\lambda / 2$ and $\lambda, \Omega_{\mathrm{L}}=0.8 \gamma(\gamma=$ $\gamma_{\mathrm{N}}+3 \mathrm{MHz}$ ), $\mathrm{V}_{\mathrm{T}}=300 \mathrm{~m} / \mathrm{s}$, pressure broadening $7.5 \mathrm{MHz} /$ Torr.

Dicke-type coherent narrowing effect is still well seen $[6,8,9]$. The further increase of buffer gas pressure (Fig.8c) results in broadening and contrast reduction of DCN dips. We should note that transmission for $L=\lambda$ in Fig. $8 \mathrm{~b}, \mathrm{c}$ is more sensitive to $\mathrm{I}_{\mathrm{L}}$ as compared with the case of $L=\lambda / 2$. Particularly, at $\mathrm{I}_{\mathrm{L}} \sim 1 \mathrm{~mW} / \mathrm{cm}^{2}$ transmission for $L=\lambda$ can be higher (the absorption is lower) than for $L=\lambda / 2$, which is caused by stronger influence of an optical pumping for $L=\lambda$. For this reason, in order to get graphs visually comparable with those presented in Fig.8 we have adjusted $\mathrm{I}_{\mathrm{L}}$ for $L=\lambda$. Fig.9 presents calculated spectra corresponding to those presented in Fig.8. An agreement with experimental results is seen. The striking point is that even with Ne pressure is up to 20 Torr the CRDN is still observable (graphs c), though less pronounced as compared with a) and b).

\section{III.2 VSOP formation in nano-cell filled with $\mathrm{Rb}$ and neon gas}

It is well-known that in the case of alkali metal vapor cells of conventional length $(0.1-10 \mathrm{~cm})$, the addition of $>0.5$ Torr of (any) buffer gas leads to disappearance of all the sub-Doppler features in the saturated absorption spectrum. Upper curve in Fig.10 shows well-known SA spectrum obtained with 3-cm long cell filled with pure Rb. The VSOP and crossover $(\mathrm{CO})$ resonances are well seen. The same spectrum recorded with $1-\mathrm{cm}$ long $\mathrm{Rb}$ cell with 6 Torr of neon is shown by the lower curve. One can see that here all the VSOP and CO resonances are completely vanished. The latter is caused by the fact that in the case of buffered cell, the velocity selectivity of $\mathrm{Rb}$ atoms reduces due to frequent collisions 
with $\mathrm{Ne}$, thus deteriorating necessary conditions to form sub-Doppler resonances.

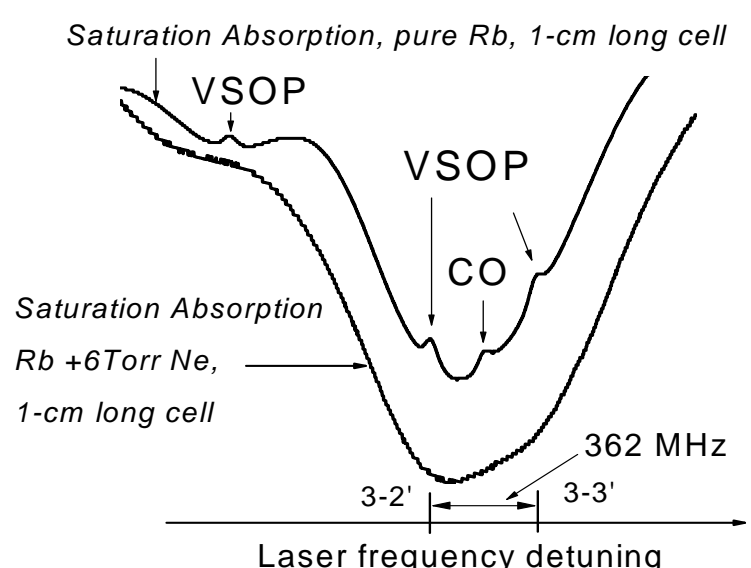

Fig.10. Upper curve is well-known SA resonances for $1-\mathrm{cm}$ long cell (pure ${ }^{85} \mathrm{Rb}, \mathrm{D}_{1}$ line), $\mathrm{CO}$ is crossover resonance; the lower curve is SA spectrum for $1 \mathrm{~cm}-$ long cell filled with $\mathrm{Rb}+6$ Torr Ne showing absence of VSOP resonances. Spectra are shifted vertically for the convenience.

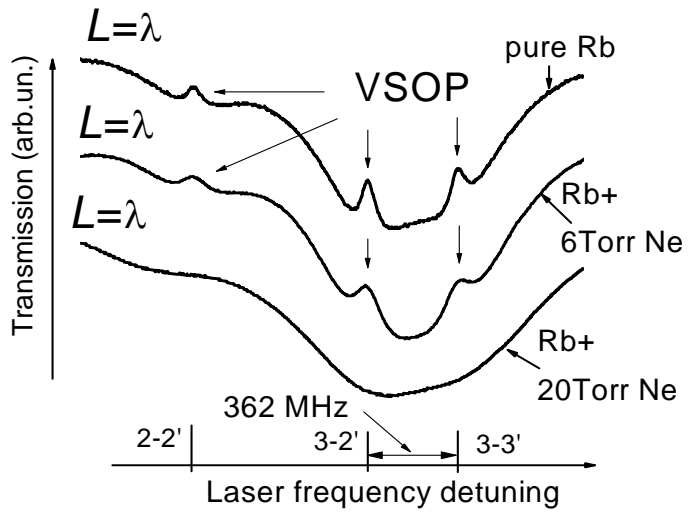

Fig.11. $\mathrm{Rb} \mathrm{D}_{1}$ line transmission spectra for $L=\lambda$. Upper curve for pure $\mathrm{Rb}$ nano-cell, VSOP resonances are well pronounced; middle curve: $\mathrm{Rb}+6$ Torr Ne nano-cell, VSOP resonances are broadened; Lower curve $\mathrm{Rb}+20$ Torr Ne, VSOP resonances are washed out. Spectra are shifted vertically for the convenience.

Fig.11 shows transmission spectra for nano-cells with $L=\lambda$ on ${ }^{85} \mathrm{Rb} \quad \mathrm{F}_{\mathrm{g}}=3 \rightarrow \mathrm{F}_{\mathrm{e}}=2,3$ and ${ }^{87} \mathrm{Rb} \quad \mathrm{F}_{\mathrm{g}}=2 \rightarrow \mathrm{F}_{\mathrm{e}}=2$ transitions $\left(\mathrm{I}_{\mathrm{L}} \sim 10 \mathrm{~mW} / \mathrm{cm}^{2}\right)$ for the following cases: upper curve for nano-cell with pure Rb: the linewidth of VSOP resonances $\sim 25 \mathrm{MHz}$; middle curve for nano-cell with Rb and 6 Torr Ne: VSOP linewidth is $\sim 90 \mathrm{MHz}$; lower curve for the nano-cell with $\mathrm{Rb}$ and 20 Torr Ne (note, VSOP resonances are absent). The comparison of Fig.6 and Fig.7 shows a striking peculiarity: the VSOPs still exist for the nano-cell with $\mathrm{Rb}+6$ Torr neon when $L=\lambda$. This important difference in behavior as compared with vapor cells of the conventional length can be explained as follows. The free path length of $\mathrm{Rb}$ atom for 6 Torr $\mathrm{Ne}$ is a bit less than $10 \mu \mathrm{m}$, so the atoms flying parallel to the windows inside the laser beam of $2 \mathrm{~mm}$ diameter experience hundreds of collisions with Ne atoms causing just additional broadening of VSOP. Meanwhile collisions of $\mathrm{Rb}$ atoms with longitudinal velocity with $\mathrm{Ne}$ atoms have a negligible impact since the thickness of nano-cell for $L=\lambda$ is less than $1 \mu \mathrm{m}$.

\section{III.3.Resonant fluorescence in nano-cell filled with $\mathrm{Rb}$ and neon gas.}
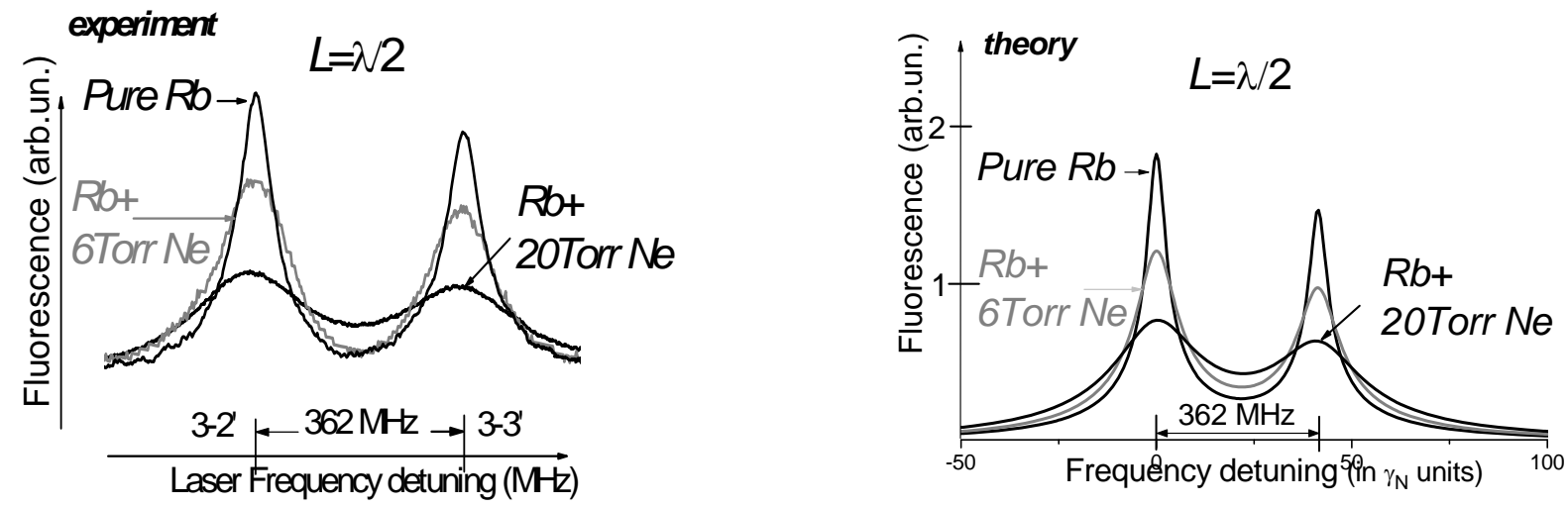

Fig.12 ${ }^{85} \mathrm{Rb} \mathrm{D}_{1}$, fluorescence spectra for $3 \rightarrow 2$ ', 3' transitions in nano-cell with $L=\lambda / 2$ for 3 cases: upper curves pure Rb: linewidth $76 \mathrm{MHz}$ (exp.) and $72 \mathrm{MHz}$ (theory); grey (middle) curves, Rb + 6 Torr Ne: $145 \mathrm{MHz}$ (exp.) and $137 \mathrm{MHz}$ (theory); lower curves , Rb +20 Torr Ne: $230 \mathrm{MHz}$ (exp) and $240 \mathrm{MHz}$ (theory). $\Omega_{\mathrm{L}}=0.4 \gamma\left(\gamma=\gamma_{\mathrm{N}}\right.$ $+3 \mathrm{MHz}), \mathrm{V}_{\mathrm{T}}=300 \mathrm{~m} / \mathrm{s}$, pressure broadening $7.5 \mathrm{MHz} /$ Torr. 
It was demonstrated in $[7,14]$ that under optimal conditions, the FWHM of the fluorescence spectrum of an individual transition for $L=\lambda / 2$ can be reduced down to $\sim 70 \mathrm{MHz}$ (in the case of ordinary cm-size cell the value is $\sim 500 \mathrm{MHz}$ ). This striking feature of the nano-cell offers an important benefit in the case of the nano-cell filled with buffer gas, too. Fig. 12 presents fluorescence spectrum when $L=\lambda / 2$ for 3 cases (left- experiment, right- theory): upper curve - nano-cell with pure $\mathrm{Rb}$ vapor; middle (grey) and lower curves - nano-cell containing $\mathrm{Rb}$ vapor +6 Torr and 20 Torr Ne, correspondingly. For theory (b): Rabi frequency $\Omega_{\mathrm{L}}=0.4 \gamma$, where $\gamma=\gamma_{\mathrm{N}}(\sim 6 \mathrm{MHz})+3 \mathrm{MHz}$ (the broadening caused by $\mathrm{Rb}-\mathrm{Rb}$ collisions at $120^{\circ} \mathrm{C}$ at the side-arm). For the calculations we used broadening rate of $\approx 7.5 \mathrm{MHz} / \mathrm{Torr}$, thus for 6 Torr, $\Gamma$ (total) $\sim 50 \mathrm{MHz}$, and for 20 Torr, $\Gamma$ (total) $\sim 150 \mathrm{MHz}$, and Maxwellian velocity distribution with $\mathrm{V}_{\mathrm{T}}=300 \mathrm{~m} / \mathrm{s}$. Laser intensity is $\sim 10 \mathrm{~mW} / \mathrm{cm}^{2}$. As it is seen, up to 20 Torr Ne the fluorescence spectrum has a sub-Doppler linewidth. As it is shown in [25], a tool for in situ pressure monitoring based on this effect can be developed.

\section{Theoretical Consideration and Discussion}

We consider a three-level atomic system (see Fig. 9) interacting with a linearly polarized laser radiation of frequency $\omega$. The three levels under the consideration are: one ground hyperfine level $F=1 \quad(|1\rangle)$ and two excited levels $F^{\prime}=2,3$ $(|2\rangle$ and $|3\rangle)$. For simplicity, we consider a one-dimensional situation, where the driving field is in the $\pm z$-direction and the atom is moving along $z$-direction with a velocity $v_{z}$. The detunings of the laser from the transitions $|1\rangle \rightarrow|2\rangle$ and $|1\rangle \rightarrow|3\rangle$ are $\Delta_{1}=\omega_{21}-\omega-k v_{z}$ and $\Delta_{2}=\omega_{31}-\omega-k v_{z}$, respectively, where $k v_{z}$ is the shift due to the Doppler effect, with $k$ being the wave vector of the excitation light. We analyze the observed spectra of resonant absorption and fluorescence on the basis of a standard density-matrix approach [?]. The dynamical behavior of the density matrix $\rho$ is given by the Liouville equation of motion

$\dot{\rho}=\frac{1}{i \hbar}\left[\hat{H}_{0}+\hat{V}, \rho\right]+$ relaxation terms,

where $H_{0}$ is the unperturbed atomic Hamiltonian, and $\hat{V}=-\hat{\mathrm{d}} \mathrm{E}(t)$ is the atom-light interaction Hamiltonian in the electric-dipole approximation with $\hat{\mathrm{d}}$ being the electric dipole operator and $E(t)$ the electric field of the radiation light. The components of the density-matrix elements of Eq. (1) can be represented by taking rotating-waveapproximation

$$
\begin{aligned}
& \dot{\rho}_{11}=-2 \operatorname{Im}\left(\Omega^{*} \rho_{21}\right)-2 \operatorname{Im}\left(\Omega^{*} \rho_{31}\right)+\gamma_{21} \rho_{22}+\gamma_{31} \rho_{33} \\
& \dot{\rho}_{22}=2 \operatorname{Im}\left(\Omega^{*} \rho_{21}\right)-2 \Gamma \rho_{22} \\
& \dot{\rho}_{33}=2 \operatorname{Im}\left(\Omega^{*} \rho_{31}\right)-2 \Gamma \rho_{33} \\
& \dot{\rho}_{21}=i \Omega_{1}\left(\rho_{11}-\rho_{22}\right)-i \Omega_{2} \rho_{32}^{*}-\left(\Gamma+i \Delta_{1}\right) \rho_{21} \\
& \dot{\rho}_{31}=i \Omega_{2}\left(\rho_{11}-\rho_{33}\right)-i \Omega_{1} \rho_{32}-\left(\Gamma+i \Delta_{2}\right) \rho_{31} \\
& \dot{\rho}_{32}=i \Omega_{2} \rho_{21}^{*}-i \Omega_{1}^{*} \rho_{31}-(2 \Gamma+i \delta) \rho_{32}
\end{aligned}
$$

where $\gamma_{i 1}$ is the rate of the spontaneous decay from the excited state $|i\rangle$ to the ground state $|1\rangle$, and $2 \Gamma=\gamma_{21}+\gamma_{1}^{\prime}=\gamma_{31}+\gamma_{2}^{\prime}$. Here $\gamma_{1}^{\prime}$ and $\gamma_{2}^{\prime}$ are the rates of population lost from the system, responsible for the optical pumping of the other ground hyperfine level with $F=2$ denoted as $\left|1^{\prime}\right\rangle$ in Fig. 9. The Rabi frequencies of the corresponding transitions are denoted by $\Omega_{1}$ and $\Omega_{2}$, respectively. As the atoms are in thermal motion, we take the average of all $\rho_{i j}$ values over the range of velocities, weighted by the one dimensional Maxwellian velocity distribution. To take into account the laser bandwidth we use the phase diffusion model of Wigner-Levy [?], in accordance with which it is assumed that the laser radiation has a Lorentzian spectrum with the Full Width at Half Maximum (FWHM) of 
$\gamma_{L}$. The bandwidth is incorporated into Eqs. (2) as a relaxation term for the non-diagonal element of the density matrix in accordance to the procedure given in [?]. The main assumptions made in the model are as follows: the atomic number

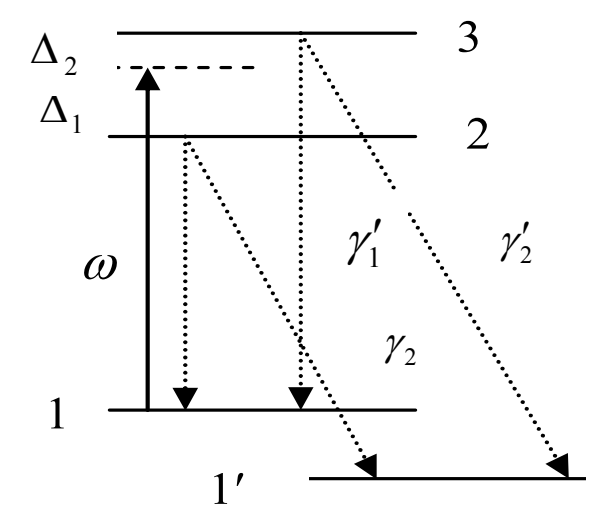

Fig. 13. The three-level scheme around the $\mathrm{D}_{1}$ line of ${ }^{85} \mathrm{Rb}$.

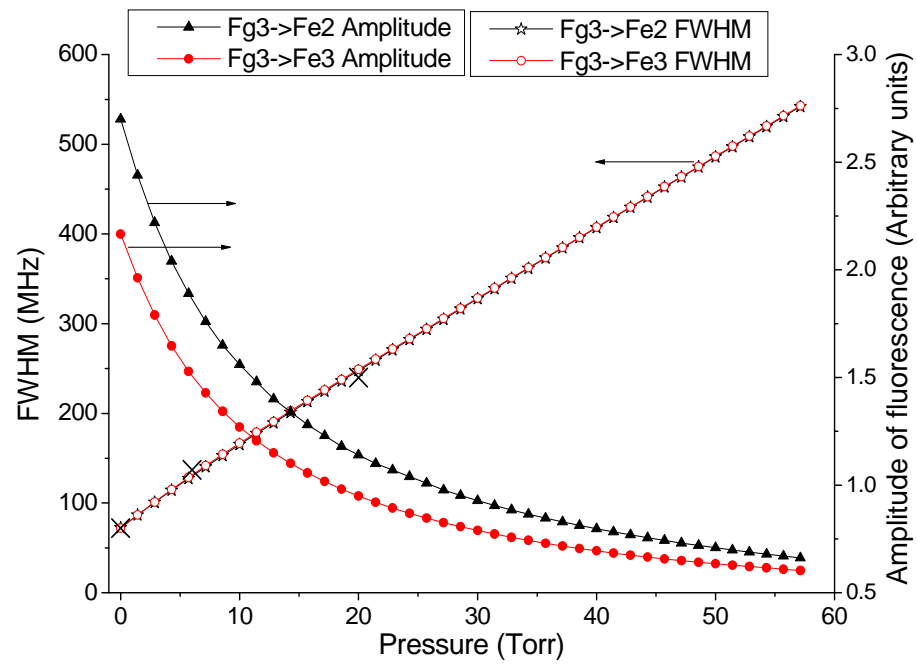

Fig.14. Dependence (theory) of the fluorescence line-width and the amplitudes for $L=\lambda / 2$ as a function of the Ne pressure for $\mathrm{Rb}, \mathrm{D}_{1}$ line, $3 \rightarrow$ $2^{\prime}, 3^{\prime}, \Omega=0.4 \gamma, \mathrm{V}_{\mathrm{t}}=300 \mathrm{~m} / \mathrm{s}$

density is assumed to be low enough so that the effect of collisions between the atoms can be ignored; the atoms experience inelastic collisions with the cell walls, i.e. atoms lose completely their optical excitation; the incident beam diameter largely exceeds the cell thickness which allows one to neglect the relaxation of atoms travelling out of the diameter of the laser beam. These assumptions allow us to take into account the collisional relaxation of each atom by solving the temporal equations for the atomic density matrix with proper boundary conditions for each atom separately. The effect of the reflection of the radiation from the highly-parallel windows of the nano-cell behaving as a Fabri-Perot cavity [5] is also taken into account. The model used here is on the lines of [5,8]. Fig. 14 presents the calculated dependence of the fluorescence line-width and the amplitudes as a function of the neon pressure for $\mathrm{Rb}, \mathrm{D} 1 \mathrm{line}, \mathrm{Fg}=$ $3 \rightarrow F e=2 ; 3, \Omega=0.4 \gamma, V_{T}=300 \mathrm{~m} / \mathrm{s}$. Note that, the FWHM data coincide for $3 \rightarrow 2$ ' and 3 $\rightarrow$ 3' spectral lines. One of the possible application using this dependence is development of an online pressure gauge which is described in [25].

\section{Electromagnetically induced transparency phenomenon; ordinary cm-size Rb cell; nano-cell filled with pure $\mathbf{R b}$; nano-cell filled with $\mathrm{Rb}$ and nitrogen gas}

The line-width of the EIT resonance in the case of small probe intensity is $\gamma_{\mathrm{EIT}} \approx 2 \Gamma_{21}+\Omega^{2} / \gamma_{\mathrm{N}}$ [17], where $\Gamma_{21}$ is the relaxation rate between ground hyperfine levels (for $\Lambda$-system), with $\Omega$ being a Rabi frequency. For the case of the nano-cell $[4,21]$, the value of $\Gamma_{21}$ is highly affected by atom-wall (cell's window) collisions. It is known that a unique collision with the dielectric surface of an uncoated vapor cell is sufficient to thermalize the ground hyperfine levels, with depolarization probability $0.5-1$. In order to preserve the atomic coherence in wall collisions either an additional gas or paraffin-coated walls were used [20]. As the size of the vapor cell is reduced, the lifetime of the ground-state coherence becomes shorter because of collisions of the atoms with the windows: $\Gamma_{21}=(2 \pi t)^{-1}$, where $t=L / u(L$ is distance between windows and $u$ is the most probable thermal velocity). Also, the EIT resonance contrast (defined as the ratio of the EIT depth to the height of shoulders of the EIT window) strongly depends on $\Gamma_{21}$. Hence, in the case of $L<1 \mu \mathrm{m}\left(2 \Gamma_{21}>100\right.$ $\mathrm{MHz}$ ), one could expect that the EIT effect will be vanished. Nevertheless, in [21,22 ] it was demonstrated that the EIT resonance can be observed in thin cell with thickness as small as $\sim 1 \mu \mathrm{m}$. The explanation is as follows: when the coupling laser is resonant with the corresponding atomic transition then the atoms moving nearly parallel to the cell windows participates in the EIT resonance formation and collisions with the windows are not essential, meanwhile when the coupling laser is detuned from the corresponding transition by a value of $\Delta$, only those atoms having the velocity 
projections $v_{\mathrm{z}}=2 \pi \Delta / k$ participate in the formation of EIT, and for these atoms the time of flight between the cell windows is small enough $\tau_{L}=L / v_{\mathrm{z}}$. This causes a frequent collisions of the atoms with the nano-cell's windows and leads to the increase of $\Gamma_{21}$ and, consequently, to the line-width broadening and contrast reduction of the EIT resonance.

One of the most dramatic different in behavior of the EIT resonance formed in ordinary cm-size Rb cell or in nano-cell filled with pure $\mathrm{Rb}$ or in nano-cell filled filled with $\mathrm{Rb}$ and nitrogen gas is dependence of the EIT resonance line-width and contrast as a function of the coupling laser frequency detuning $\Delta$ (from the corresponding atomic transition) which is demonstrated below. The experimental arrangement is sketched in Fig 15. Both beams ( $\varnothing 3 \mathrm{~mm})$ of two single-frequency extended cavity diode lasers (ECDL) (coupling and probe) with $\lambda \approx 794 \mathrm{~nm}$ (the linewidth is $\sim 1$

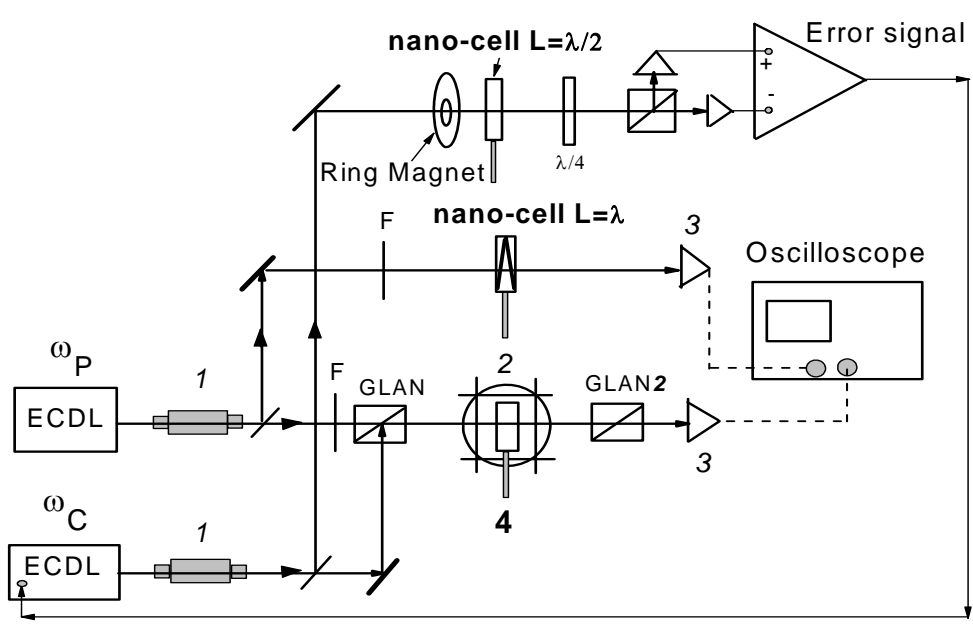

Fig. 15 a) Sketch of the experimental Set-Up. (1)-are Faraday isolators, (2)- Helmholts coils , (3)- photodiodes , (4)- CCL; nano-cell filled with pure $\mathrm{Rb}$; and nano-cell filled with $\mathrm{Rb}$ and nitrogen gas.

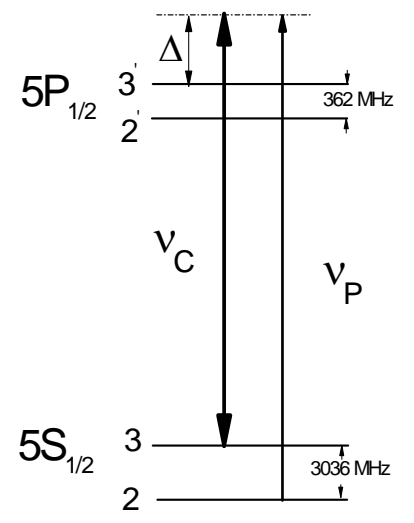

Fig. 15 b) energy levels of the ${ }^{85} \mathrm{Rb} \mathrm{D}_{1}$ line involved in EIT process. The coupling is shifted by $\Delta$ in blue side from 3-3' transition, while the probe frequency is scanning across $2 \rightarrow 3$, transition.

$\mathrm{MHz}$ ) are well superposed and directed onto the cell (4) at near-normal incidence with the help of the first Glan prism (the coupling and probe beams have linear and perpendicular polarizations). The second Glan 2 prism has been used, which allows to provide separate detection of the spectra of the probe and coupling lasers, (1)-are Faraday isolators. Thin cell (4), of nano-metric thickness, was placed inside the three pairs of mutually perpendicular Helmholts coils (2) providing possibility to cancel laboratory magnetic field as well as to apply homogeneous magnetic field. The optical radiations were recorded by the photodiodes (3) and the signal of the photodiodes was intensified and recorded by a twochannel digital storage oscilloscope Tektronix TDS 3032B, F-are filters. An improved Dichroic-Atomic-Vapor-LaserLocking (DAVLL) method, realized in a separate nano-cell with the thickness $L=\lambda / 2$, is used for the coupling laser frequency stabilization; this technique is presented in [23]. The frequency reference spectra formation has been realized with the help of an auxiliary nano-cell with thickness $L=\lambda[4]$.

Dependence of the EIT resonance line-width and amplitude (contrast) as a function of the coupling detuning $\Delta$ for $6 \mathrm{~cm}$-long cell is shown in Fig 16. The coupling power is $\sim 10 \mathrm{~mW}$, while the probe power is $\sim 20 \mu \mathrm{W}$. As it is seen when $\Delta$ is increasing : $\Delta_{0}, \Delta_{1}, \Delta_{2}$, values are 0,90 and $120 \mathrm{MHz}$, correspondingly, the line-width of EIT resonances is slightly reduced and line-width values (FWHM)are 13.5, 12.7 and $12.5 \mathrm{MHz}$, correspondingly, while the amplitude of the EIT resonances remain practically the same. Some reduction of the EIT amplitude with $\Delta$ increasing is caused by Maxwellian velocity distribution. VSOP peak is demonstrating increasing of the absorption, since the coupling laser transfer a number of atoms from the level 3 to level 2 , and this causes increase of the probe absorption for $2 \rightarrow 2$ ', 3' transitions (the EIT resonance is superimposed with the VSOP at 2- 3'transition ). Note that physical nature of this VSOP is different to the another VSOP presented in Fig.7. Dependence of the EIT resonance line-width and amplitude as a function of the blue detuning $\Delta$ of the coupling for thickness $L=\lambda$ is shown in Fig $17 . \mathrm{P}_{\mathrm{C}} \sim 15 \mathrm{~mW}, \mathrm{P}_{\mathrm{P}} \sim 20 \mu \mathrm{W}$, nanocell's reservoir temperature is $120^{\circ} \mathrm{C}$. As it is seen when $\Delta$ is increasing : $\Delta_{0}, \Delta_{1}, \Delta_{2}, \Delta_{3}, \Delta_{4}$ values are $0,15,40,50$ and 85 $\mathrm{MHz}$, correspondingly, the line-width of EIT resonances are rapidly increasing and line-width values are $30,33,51$ and 
$74 \mathrm{MHz}$, correspondingly, while the amplitude of the EIT resonances are rapidly reduced. The physical explanation of this fact is presented above.

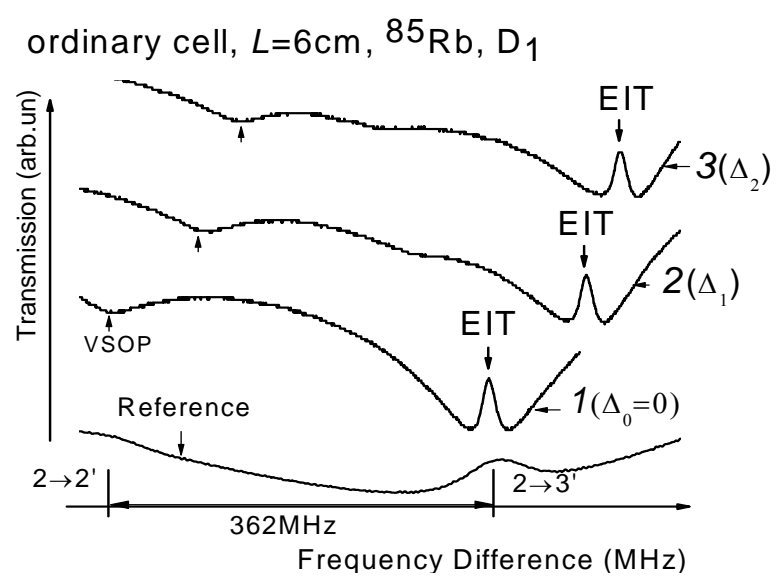

Fig.16.Tansmission spectra of the probe when the coupling frequency is shifted by $\Delta$ with the respect of 3 $\rightarrow 3$ ' transition, $\Delta_{0}, \Delta_{1}, \Delta_{2}$ are equal to : $0 ; 90$ and 120 $\mathrm{MHz}$, for the curves $1,2,3$, for $6 \mathrm{~cm}$-long cell . Lower curve is the reference spectrum which is formed with the help of an auxiliary nano-cell with thickness $L=\lambda$. For the VSOP peak see the text. Spectra are shifted vertically for the convenience.

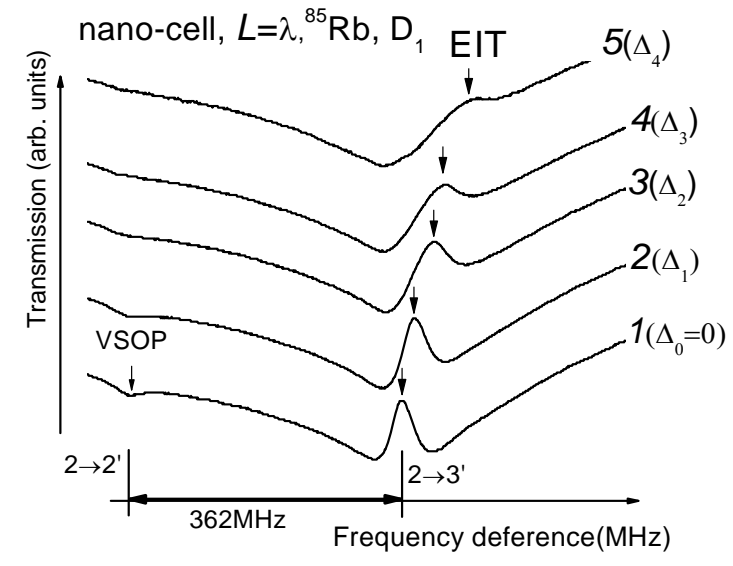

Fig.17. Nano-cell filled with pure $\mathrm{Rb}$, transmission spectra of the probe when the coupling frequency is shifted by $\Delta$ with the respect of $3 \rightarrow 3$, transition, $\Delta_{0}$, $\Delta_{1}, \Delta_{2}, \Delta_{3}, \Delta_{4}$ values are $0,15,40,50$ and $74 \mathrm{MHz}$, correspondingly, for the curves $1,2,3,4,5$, nano-cell thickness $L=\lambda$. EIT resonances are indicated by the vertical arrows. For the VSOP peak see the text. Spectra are shifted vertically for the convenience.

to study atom-walls collisions, i.e. a wall's material. Note, that that it is expected that a high temperature of the nano-cell wall $>400{ }^{\circ} \mathrm{C}$ may causes a reduction of the depolarization probability due to atom-wall collision, i.e. smaller dependence of the EIT line-width versus the coupling detuning could be observed in this case. Thus, there is a dramatic different in behavior of the EIT resonance in nano-cell filled with pure Rb and CCL. Comparison of the EIT resonance line-width for the both cases vs the coupling detuning is shown in Fig.18. Note, that increasing of the $\Delta$ in the case of nano-cell is causes rapid increase of the line-width, meanwhile for CCL even a small reduction of the line-width is observable.

It is expected that addition of a buffer gas (under the appropriate pressure) inside the nano-cell will reduce dramatic behavior of the EIT resonance contrast and the line-width as a function of coupling detuning. If mean free path for

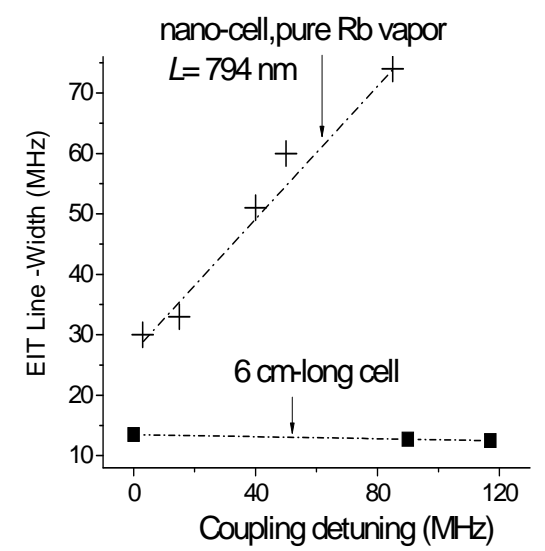

Fig.18. Dependence of the EIT resonance line-width vs the coupling detuning $\Delta$, upper curve - for the nano-cell filled with pure $\mathrm{Rb}, L=794 \mathrm{~nm}$, lower curve - for 6-cm long Rb cell. Dot-lines are for eyes guide.

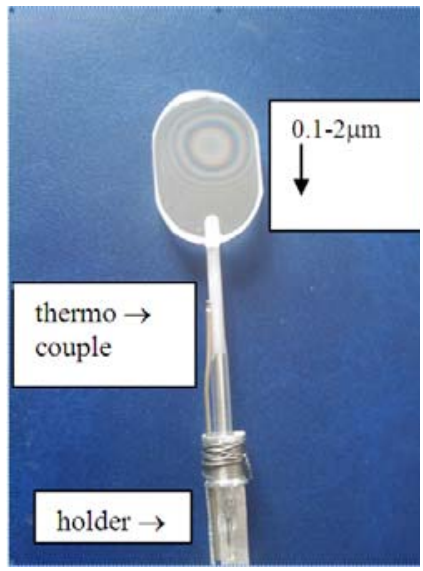

Fig.19. Photo of the $\mathrm{Rb}$ nano-cell filled with $\mathrm{N}_{2}$. Thermocouple is tied to the sapphire side-arm for the $\mathrm{T}$ measurement at the top of the $\mathrm{Rb}$ metal column [3].The nano-cell is fixing inside the oven with the help of the aluminum holder. 
Rb-buffer collisions will be much less than the distance L between nano-cell's windows ( $L=\lambda \sim 800 \mathrm{~nm}$ ) this will prevent direct atom-walls collisions, thus, this will avoid increase of $\Gamma_{21}$ (and, consequently, avoiding the strong line-

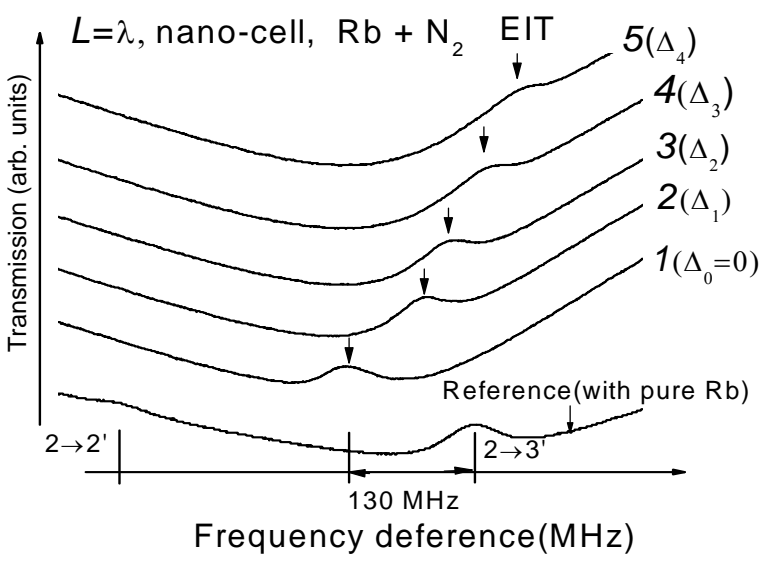

Fig.20. Nano-cell filled with $\mathrm{Rb}$ and $\mathrm{N}_{2}$, transmission spectra of the probe when the coupling frequency is shifted by $\Delta_{0} \Delta_{1} \Delta_{2} \Delta_{3} \Delta_{4}$ which are equal to $0 ; 79,98.5,142$ and $177 \mathrm{MHz}$,correspondingly , for the curves 1,2,3,4,5, thickness $L=\lambda$. EIT resonances are indicated by vertical arrows. Reference spectrum is formed by the help of an auxiliary nano-cell filled with pure $\mathrm{Rb}$ and thickness $\mathrm{L}=\lambda$. Spectra are shifted vertically for the convenience.

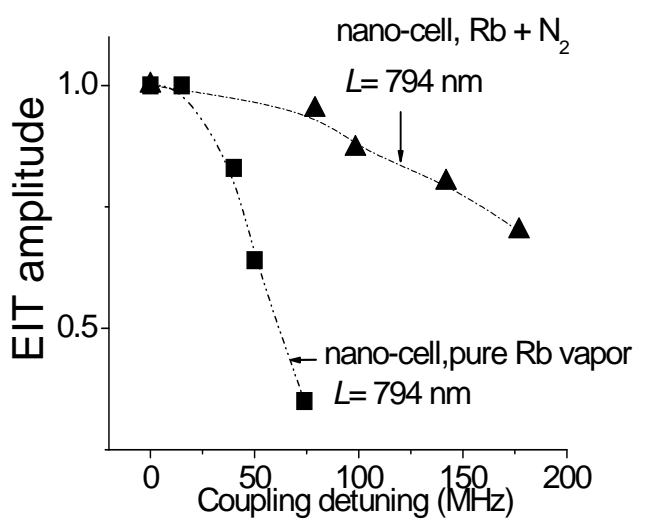

Fig.21. Dependence of the EIT resonance amplitude as a function of the coupling-laser detuning $\Delta$, upper curve - the nano-cell, $\mathrm{Rb}+\mathrm{N}_{2}$ gas, $L=794 \mathrm{~nm}$, lower curve -nano-cell, pure Rb, $L=794 \mathrm{~nm}$. Dot-lines are for eyes guide

width broadening and contrast reduction).In order to check this statement a special nano-cell filled with the Rb and nitrogen $\left(\mathrm{N}_{2}\right)$ with the pressure $\sim 20$ Torr was fabricated and is shown in Fig 19. The gap has wedged form in the vertical direction from $100 \mathrm{~nm}$ (centum of the ring part) up to $2 \mu \mathrm{m}$ near the bottom. Collisional cross section of Rb- $\mathrm{N}_{2}$ by several times is larger than that of $\mathrm{Rb}-\mathrm{Ne}$, thus providing smaller mean free path for $\mathrm{Rb}-\mathrm{N}_{2}$ collisions (mean free path $\sim$ $300 \mathrm{~nm}$ ). Dependence of the EIT resonance line-width and amplitude as a function of the blue detuning $\Delta$ of the coupling for nano-cell filled with $\mathrm{Rb}$ and 20 Torr $\mathrm{N}_{2}$ with thickness $L=\lambda$ is shown in Fig 20. As it is seen when $\Delta$ is increasing, i.e. $\Delta_{0}, \Delta_{1}, \Delta_{2}, \Delta_{3}, \Delta_{4}$ are equal to $0 ; 79,98.5,142$ and $177 \mathrm{MHz}$,correspondingly, the EIT amplitude reduction is much weaker than that in the case of the nano-cell filled with pure Rb vapor. Reference spectrum is formed by the help of an auxiliary nano-cell filled with pure $\mathrm{Rb}$ and with thickness $\mathrm{L}=\lambda$, thus showing atomic transition position not perturbed by $\mathrm{N}_{2}$ collisions , while EIT resonance for $\Delta_{0}=0$ is red shifted by $130 \mathrm{MHz}$ which means that atomic transition $3 \rightarrow 3^{\prime}$ is red shifted due to $\mathrm{Rb}-\mathrm{N}_{2}$ collisions. The comparison of the EIT resonance amplitude for the both cases versus the coupling detuning is shown in Fig.21. Thus, use of buffer gas $\mathrm{N}_{2}$ strongly extended the region of the coupling detuning where EIT resonances is still possible to create.

\section{Conclusions}

Comparison of the resonant absorption in the nano-cell filled with $\mathrm{Rb}$ vapor with another nano-cell filled with $\mathrm{Rb}$ and neon gas with pressure 6 Torr and 20 Torr shows that the spectra of the resonant absorption demonstrate subDoppler narrowing for the thickness $L=\lambda / 2$ and broadening for the thickness $L=\lambda$, thus manifesting the effect of collapse and revival of Dicke-type narrowing ( $\lambda=794 \mathrm{~nm}$ is a resonant laser wavelength). In the Rb cells of the conventional length filled with buffer gas, in the saturated absorption VSOP resonances are washed out when pressure $>0.5$ Torr. In contrast, for the nano-cell with $L=\lambda$ the VSOP resonances located at the atomic transition are still observable even when neon pressure is $\geq 6$ Torr. Narrowband fluorescence spectra emitted by the nano-cell with $L=\lambda / 2$ can be used as a convenient tool for online buffer gas pressure monitoring in the MRC in its several centimeter-long part, for the conditions when ordinary pressure gauges are unusable. Developed theoretical model well describes all experimentally observed peculiarities.A strong increasing of the EIT resonance line-width and contrast (amplitude) reduction for a large detuning of the coupling laser frequency with respect to the corresponding transition is revealed in the nano-cell filled 
with pure $\mathrm{Rb}$ and thickness $L=\lambda$. It is shown that this is completely in contradiction to that observable in the case of CCL filled with the $\mathrm{Rb}$. It is demonstrated that addition of buffer gas nitrogen (under the appropriate pressure) strongly extended the region of the coupling detuning where EIT resonance is still possible to create with the help of the nanocell with the thickness $L=\lambda$.

\section{Acknowledgements}

The authors thank A. Sarkisyan for his valuable participation in the fabrication of the MRC. Research conducted in the scope of the International Associated Laboratory IRMAS. Armenian team thanks for the ANSEF PS 1868 support. Armenian team thanks also for the Grant INTAS 06-1000017-9001 (prolonged by Armenian government).

\section{References}

[1]. S. Briaudeau, D. Bloch, M. Ducloy, "Sub-Doppler spectroscopy in a thin film of resonant vapor", Phys. Rev. A 59, (1999) 3723 and references therein.

[2].A.Ch. Izmailov, Opt. and Spectr.74 (1), 25 (1993).

[3]. D.Sarkisyan, D.Bloch, A.Papoyan, M.Ducloy, "Sub-Doppler spectroscopy by sub-micron thin Cs- vapor layer" Opt. Commun. 200, 201 (2001).

[4]. D. Sarkisyan, A. Papoyan, "Optical processes in micro- and nanometric thin cells containing atomic vapor" in New Trends in Quant. Coher. and Nonl. Opt., R. Drampyan ed. ( Nova Sc. Publ., 2009).

[5]. G. Dutier, S. Saltiel, D. Bloch, M. Ducloy, "Revisiting optical spectroscopy in a thin vapour cell: mixing of reflection and transmission as a Fabry- Perot microcavity effect," J. Opt. Soc. Am. B 20, 793-800 (2003).

[6]. G.Dutier, A.Yarovitski, S.Saltiel, A. Papoyan, D. Sarkisyan, D. Bloch, M. Ducloy, "Collapse and revival of a Dicketype coherent narrowing in a sub-micron thick vapor cell transmission spectroscopy" Europhys. Lett. 63(1),35 (2003).

[7]. D.Sarkisyan, T. Becker, A.Papoyan, P. Thoumany, H.Walther, "Sub-Doppler Fluorescence on atomic $\mathrm{D}_{2}$ line of Sub-Micron Rubidium Vapor Layer", Applied Physics B 76, 625-631(2003).

[8]. G. Nikogosyan, D. Sarkisyan, Yu. Malakyan, "Resonant absorption and fluorescence of atomic layer of the thickness of the order of light wavelength", J. of Opt. Tech. 71, 602 (2004).

[9]. D. Sarkisyan, T. Varzhapetyan, A. Sarkisyan, Yu. Malakyan, A. Papoyan, A. Lezama, D. Bloch, M.Ducloy, "Spectroscopy in an Extremely Thin Vapour Cell: Comparing the Cell Length Dependence in Fluorescence and in Absorption Techniques," Phys. Rev. A 69, 065802 (2004).

[10]. C. Andreeva, S. Cartaleva, L. Petrov, S. M. Saltiel, D. Sarkisyan, T. Varzhapetyan, D. Bloch, M. Ducloy, "Saturation effects in the sub-Doppler spectroscopy of cesium vapor confined in an extremely thin cell," Phys. Rev. A 76, 013837 (2007).

[11]. T. Varzhapetyan, A. Nersisyan, V. Babushkin, D. Sarkisyan, S. Vdovi'c, G. Pichler, "Study of atomic transition self-broadening and shift with the help of a nano-cell," J. Phys. B: At. Mol. Opt. Phys. 41, 185004 (2008).

[12]. A. Sargsyan, D.Sarkisyan, Y. Pashayan-Leroy, C. Leroy, P. Moroshkin, A. Weis "Dependence of the Cs absorption $\mathrm{D}_{1}$ linewidth on the thickness of atomic vapor column", Journal of Contemporary Physics, 43, 7 (2008).

[13]. S. Cartaleva, S. Saltier, A. Sargsyan, D. Sarkisyan, D. Slavov, P. Todorov, K. Vaseva, "Sub-Doppler spectroscopy of cesium vapor layers with nanometric and micrometric thickness," J. Opt. Soc. Am. B 26, 1999-2006 (2009).

[14]. A. Sargsyan, G. Hakhumyan, A. Papoyan, D. Sarkisyan, A. Atvars, M. Auzinsh, "A novel approach to quantitative spectroscopy of atoms in a magnetic field and applications based on an atomic vapor cell with $L=\lambda$ ”, Appl. Phys. Lett., 93, 021119 (2008).

[15].G. Hakhumyan, D. Sarkisyan, A. Sargsyan, A. Atvars, and M. Auzinsh "Investigation of Rb $D_{1}$ Atomic Lines in Strong Magnetic Fields by Fluorescence from a Half-Wave-Thick Cell “Opt. and Spectrosc., 108 , 685 (2010).

[16]. David Sarkisyan and Aram Papoyan "Formation of Narrow Optical Resonances using submicron - thin atomic vapor layer" Invited, Modern Optics and Photonics. Atoms and Structured Media pp. 257-288, World Scientific Publishing Co., Singapore (2010).

[17]. R. Wynands, A. Nagel, "Precision spectroscopy with coherent dark states", Appl. Phys. B:Lasers Opt. 68, 1 (1999).

[18]. D. Petrosyan, Yu. Malakyan, "Electromagnetically induced transparency in a thin vapor film”, Phys. Rev. A 61, 053820-053828 (2000).

[19].M. Fleischhauer, A. Imamoglu, J. P. Marangos "Electromagnetically induced transparency "Optics in Coherent 
Media” Rev. Modern Physics,.77, pp.633-674 (2005).

[20]. S. Knappe, L. Hollberg, J. Kitching, ”Dark-line atomic resonances in submillimeter structures”, Opt. Lett. 29, 388$390(2004)$

[21]. A.Sargsyan, D.Sarkisyan, A.Papoyan, "Dark-line atomic resonances in sub-micron thin Rb vapor layer", Phys. Rev. A 73, 033803-033810 (2006).

[22]Y. Pashayan-Leroy,C. Leroy, A Sargsyan, A. Papoyan, D. Sarkisyan,”Electromagnetically induced transparency: the thickness of the vapor column is of the order of a light wavelength ", J. Opt. Soc. Am. B 24, 1829(2007).

[23].A. Sargsyan, A.V. Papoyan, D. Sarkisyan, A. Weis "Efficient technique for measuring laser frequency stability" Europ.Phys. Journ. Appl. Physics (EPJ AP) 48, 20701 (2009).

[24] .T. Baluktsian, C. Urban, T. Bublat ,H.Giessen, R. Löw and T. Pfau, "Fabrication method for microscopic cells for alkali atoms "Optics Lett. 35, 1950, 2010.

[25].G. Hakhumyan, A. Sargsyan, C.Leroy, Y. Pashayan-Leroy, A.Papoyan, D. Sarkisyan "Essential Features of Optical Processes in Rb submicron Thin Cell Filled with Neon Gas" Optics Express, 18, 14577 (2010).

[26]. S. Stenholm, Foundations of Laser Spectroscopy (New York: Wiley, 1983).

[27]. J.H. Eberly, "Atomic Relaxation in the Presence of Intense Partially Coherent Radiation Fields," Phys. Rev. Lett. 37, 1387-1390 (1976).

[28]. G.S. Agarwal, "Exact Solution for the Influence of Laser Temporal Fluctuations on Resonance Fluorescence," Phys. Rev. Lett. 37, 1383-1386 (1976).

[29]. G.S. Agarwal, "Quantum statistical theory of optical-resonance phenomena in fluctuating laser fields," Phys. Rev. A 18, 1490-1506 (1978). 